\title{
Prospects for rare B-decays at the $\mathrm{LHCb}$
}

\author{
Nicola Serra ${ }^{a}$ \\ Nationaal instituut voor subatomaire fysica (NIKHEF), Science Park 1051098 XG \\ Amsterdam , The Netherlands \\ Abstract.The search for physics beyond the Standard Model will be the primary \\ task of the LHC experiments. At present, the most stringent constraints to new \\ physics are given by Flavor Changing Neutral Current (FCNC) decays, such as \\ processes that involve $b \rightarrow s \gamma$ transitions. The LHCb experiment has the possibility \\ to consistently improve present results on rare FCNC decays of B-mesons. Here, \\ the $\mathrm{LHCb}$ potential for the search of new physics in the rare decays $B_{s} \rightarrow \mu^{+} \mu^{-}$, \\ $B_{d} \rightarrow K^{*} l^{+} l^{-}, B_{s, d} \rightarrow e^{ \pm} \mu^{\mp}$ and $B_{s} \rightarrow \phi \gamma$ is discussed.
}

\section{Introduction}

Rare decays of B-mesons which proceed via Flavor Changing Neutral Currents (FCNC) are forbidden at the tree level and suppressed by the GIM mechanism in the Standard Model (SM). As a result, new physics (NP) contributions can enter at the same order as SM contributions. Moreover, since new particles contribute as virtual particles, the potential of discovering NP is not limited by the available energy, but only by our ability to make precise predictions and measurements. In particular FCNC involving $b \rightarrow d, s$ transitions are excellent indirect probes for NP, providing information about the masses and couplings of virtual NP particles entering into the loops. Here the decays $B_{s, d} \rightarrow \mu^{+} \mu^{-}$, $B_{d} \rightarrow K^{*} l^{+} l^{-}, B_{s} \rightarrow \phi \gamma$ and $B_{s, d} \rightarrow e^{ \pm} \mu^{\mp}$ will be discussed. For all these decays the $\mathrm{LHCb}$ experiment [1] has the possibility to significantly improve the results of current experiments.

\section{The rare decays: $B_{s} \rightarrow \mu^{+} \mu^{-}$and $B_{s, d} \rightarrow e^{ \pm} \mu^{\mp}$}

The decay $B_{s} \rightarrow \mu^{+} \mu^{-}$is a very rare decay. Its branching ratio is predicted to be $(3.35 \pm 0.32) \times 10^{-9}$ within the SM [2]. However, this observable is quite sensitive to NP involving new scalar/pseudo-scalar couplings. For instance models involving an extended Higgs sector, such as SUSY models, will change this branching ratio. In the Minimal Supersymmetric extension of the SM (MSSM) this branching ratio depends on the sixth power of the parameter $\tan \beta$. The decay $B_{s} \rightarrow \mu^{+} \mu^{-}$has not yet been observed, and will be one of the key measurements for the $\mathrm{LHCb}$ experiment. Its most recent upper limit is $3.6 \times 10^{-8} @ 90 \% C L$, set by the Tevatron experiments [3].

The LHCb event selection for this decay is based on a "soft" preselection to reject most of the background, followed by a multidimensional analysis based on three variables: the invariant mass of the di-muons, a geometrical likelihood and a particle identification likelihood. The geometrical likelihood combines

\footnotetext{
${ }^{a}$ e-mail: nicola.serra@cern.ch
} 

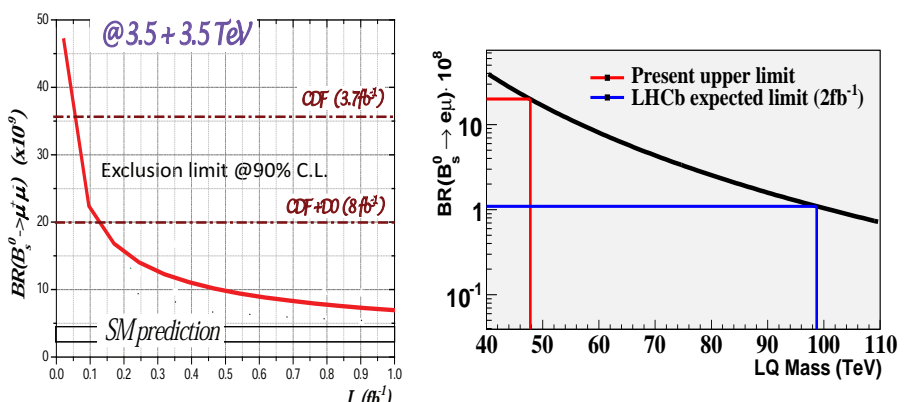

Figure 1: Exclusion limit at 90\% CL for the measurement of $B R\left(B_{s} \rightarrow \mu^{+} \mu^{-}\right)$at $\mathrm{LHCb}$ (left). Exclusion limit for the $B R\left(B_{s} \rightarrow e^{ \pm} \mu^{\mp}\right)$ and corresponding limit for the Pati-Salam Leptoquark (right).

topological discriminating variables. For a given number of observed events the exclusion at $90 \% C L$ or the $3 \sigma$ signal evidence ( $5 \sigma$ discovery) can be computed. This method is described in [4]. In Fig.1 the expectation for the measurement of the $\operatorname{Br}\left(B_{s} \rightarrow \mu^{+} \mu^{-}\right)$at LHCb, as a function of the integrated luminosity, is shown. The $\mathrm{LHCb}$ experiment will therefore be able of scanning the interesting region from the final Tevatron limit down to the SM expectation with the first two years of data taking in 2010 and 2011, corresponding to an integrated luminosity of $1 \mathrm{fb}^{-1}$.

A similar analysis strategy is foreseen for the rare decays $B_{s, d} \rightarrow e^{ \pm} \mu^{\mp}$. These decays are forbidden in the SM. The simplest model which allows these decays is the Pati-Salam model, based on the $S U(4)$ symmetry group [5]. In this model the lepton number is seen as the forth color of the theory. LHCb will further constrain the $B R(B \rightarrow e \mu)$ which in turn will increase the lower limit on the mass of the Pati-Salam leptoquark by a factor 2 to $100 \mathrm{TeV}$ (Fig. 1) [6].

\section{Angular observables in the $B_{d} \rightarrow K^{*} \mu \mu$ decay}

The decay $B_{d} \rightarrow K^{*} \mu \mu$ is fully described by three angles $\left(\theta_{l}, \theta_{k}, \phi\right)[9]$ and the di-muon invariant mass $q^{2}$. The angular distribution of this decay gives access to a number of observables sensitive to NP [8]. The most promising of these observables for the search of NP is the Forward-Backward Asymmetry $A_{F b}$. In particular the zero-crossing point of this asymmetry is well predicted in the SM and in a number of NP extensions of the SM. Recently, measurements of the $A_{F B}$ were published by the BaBar, Belle and CDF experiments [7]. Although the statistics is too low for claiming for an evidence of NP, all the measurements show no evidence of a zero-crossing point, a distinctive sign of the SM. The expected sensitivity for $\mathrm{LHCb}$ to the $A_{F B}$ for $2 \mathrm{fb}^{-1}$ of data taking, assuming the SM, is shown in Fig.2. The expected resolution in the zero-crossing point 
is expected to be $\sigma\left(s_{0}\right)=0.5 \mathrm{GeV}^{2}[9]$.

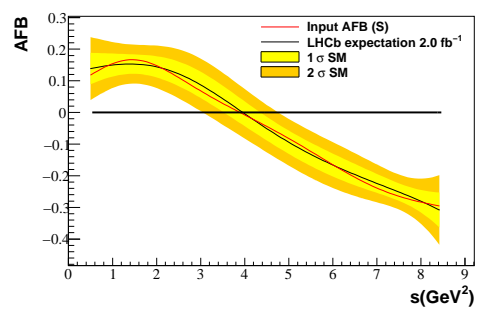

Figure 2: Expectation for the measurement of the $A_{F B}$ at $\mathrm{LHCb}$ for $2 \mathrm{fb}^{-1}$ (right).

\section{Measuring the polarization of the photon in $b \rightarrow s \gamma$ processes}

One of the most stringent constraint on NP comes from measurements of $b \rightarrow s \gamma$ transitions [10]. In the SM the emitted photon is almost completely left-handed polarized due to the V-A structure of the SM. The right-handed part is of the order of $\frac{m_{s}}{m_{b}} \sim 10^{-2}$. However NP contributions (as shown in the right diagram of Fig. 3), could significantly enhance the right-handed contribution. At the $\mathrm{LHCb}$, the helicity of the photon can be probed with the decays $B_{s} \rightarrow \phi \gamma$ and $B_{d} \rightarrow K^{*} e^{+} e^{-}$. In the former decay the photon helicity can be extracted by performing a time dependent analysis. The decay rate can be written, for $B_{s}$ and $\bar{B}_{s}$ respectively:

$$
\begin{aligned}
B(\bar{B})(t) & =B_{0} e^{-\Gamma t}\left(\cosh \left(\frac{\Delta \Gamma t}{2}\right)-\right. \\
& \left.-H \sinh \left(\frac{\Delta \Gamma t}{2}\right) \pm C \cos \left(\Delta m_{s} t\right) \mp S \sin \left(\Delta m_{s} t\right)\right)
\end{aligned}
$$

where $H \sim(2 \psi)$ and $\tan \psi=\frac{A_{R}}{A_{L}}$. It is worth noticing that for measuring the parameters $C$ and $S$ the knowledge of the initial flavor of the B-meson is needed. To measure the parameter $H$, instead, no flavor tagging information is necessary, with a clear experimental advantage [11]. The LHCb sensitivity for this decay in a nominal year of data taking $\left(2 \mathrm{fb}^{-1}\right)$, according to the MonteCarlo analysis, is $\sigma\left(\frac{A_{R}}{A_{L}}\right) \sim 0.1[9]$.

Another way for accessing the photon polarization in $b \rightarrow s \gamma$ transition is by measuring the virtual photon in the $B_{d} \rightarrow K^{*} e^{+} e^{-}$decay. The region of low di-lepton invariant mass, where the photon is quasi-real is dominated by the left diagram of Fig. 3 in the SM. The polarization of the photon is here accessible by performing an angular analysis.

According to MC simulation a sensitivity of $\frac{2 A_{R}}{A_{L}} \sim 0.1$ is expected in a nominal year of data taking [12]. A similar sensitivity on the right-handed 

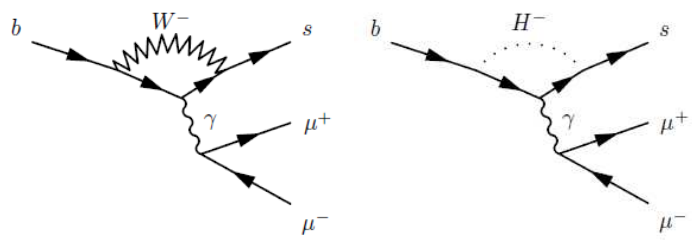

Figure 3: Possible diagrams for the $B_{s} \rightarrow K^{*} l^{+} l^{-}$decays, for the SM (left) and for a NP model (right).

component is expected for these two uncorrelated measurements, which allows for an independent cross-check.

\section{References}

[1] A. Augusto Alves Junior et al. [LHCb collaboration], J.Instr. 3 4S08005 (2008).

[2] M. Blanke et al., JHEP 003, 0610 (2006).

[3] T. Aaltonen et al. [CDF collaboration], CDF note 9892 (2009). D collaboration, D note 5344-CONF (2007).

[4] D. Martnez, J.A. Hernando and F. Teubert, LHCb note 2007-033 (2007).

[5] J.Pati and A.Salam, Phys. Rev. D 10275 (1974).

[6] N. Serra and W. Bonivento, LHCb note 2007-028 (2007).

[7] B. Aubert et al. [Babar collaboration], Phys.Rev. D 73, 092001 (2006). J.T. Wei et al. [Belle collaboration] Phys.Rev.Lett. 103171801 (2009). T. Aaltonen et al., Phys.Rev. D 79, 011104(R) (2009).

[8] W. Altmannshofer et al., JHEP 0901, 019 (2009). F. Kruger and J. Matias, Phys. Rev. D71, 094009 (2005). U. Egede et al, JHEP 032, 0811 (2008).

[9] B. Adeva et al, [LHCb collaboration], arXiv:0912.4179v2.

[10] K. Fujikawa and A. Yamada Phys. Rev. 49, 5890 (1994). J. Ellis et al., JHEP 063, 0605 (2006).

[11] F. Muheim, Y. Xie, R. Zwicky, Phys.Lett. B 664, 174-179 (2008)

[12] J. Lefranois, M.H. Schune, LHCb note 2009-008 (2009). 